\title{
Synergistic effects of lovastatin and celecoxib on caveolin-1 and its down-stream signaling molecules: Implications for colon cancer prevention
}

\author{
SURESH GURUSWAMY and CHINTHALAPALLY V. RAO \\ Department of Medicine, Hem-Onc Section, University of Oklahoma \\ Cancer Institute, OUHSC, Oklahoma City, OK 73104, USA
}

Received February 17, 2009; Accepted April 15, 2009

DOI: 10.3892/ijo_00000418

\begin{abstract}
Progression of colon cancer is associated with the up-regulation of cyclooxygenase-2 (COX-2) and hydroxymethyl glutaryl CoA reductase (HMG-R). Clinical and preclinical evidence shows that a combination of COX-2 and HMG-R inhibitors provide additive/synergistic chemopreventive effects against colorectal cancer. However, the mechanism by which statins and NSAIDs inhibit cancer growth is not yet fully understood. We aimed to identify critical molecules and signal pathways modulated by a combination of lovastatin and celecoxib in the human HCT-116 colon cancer cell line. HCT-116 cells were exposed to $50 \mu \mathrm{M}$ celecoxib, $25 \mu \mathrm{M}$ lovastatin or a combination of both to assess their effect in modulating caveolin-1 expression and its down-stream signaling pathways. Our results suggest that a combination of lovastatin and/or celecoxib suppressed caveolin-1 expression and membrane localization profoundly when compared to either agent alone. Lovastatin and/or celecoxib also inhibited caveolin-1-dependent cell survival signals mediated through Akt activation as well as its down-stream effectors such as phosphorylated ERK and STAT3 in HCT-116 cells. Treatment with lovastatin or celecoxib decreased the levels of cyclin D1, CDK2, pRb and E2F1, while the combination treatment showed more pronounced suppression. In addition, lovastatin and celecoxib also decreased the amount of
\end{abstract}

Correspondence to: Dr Chinthalapally V. Rao, 975 NE 10th Street, BRC Room \#1203, OU Cancer Institute, University of Oklahoma Health Sciences Center, Oklahoma City, OK 73104, USA

E-mail:cv-rao@ouhsc.edu

Abbreviations: COX-2, cyclooxygenase-2; HMG-R, HMG-CoA reductase; ERK, extracellular signal-regulated kinase; CDK2, cyclin-dependent kinase; STAT3, signal transducer and activator of transcription 3; cav-1, caveolin-1

Key words: colon cancer, chemoprevention, caveolin-1, cyclooxygenase-2, hydroxy methyl glutaryl CoA, reductase, tumor suppressor proteins, apoptosis cholesterol rich cytoplasmic lipid bodies (storehouses of esteridied arachidonates) by $80 \%$, while the combination showed a complete inhibition. Overall, our data suggest that a combination of COX-2 and HMG-R inhibitors synergistically inhibits caveolin-1 and its associated signaling pathways.

\section{Introduction}

Colon cancer is one of the leading causes of cancer-related death in the Western world, including the United States. According to the American Cancer Society, 112,340 new cases of colon cancer will be diagnosed with 52,180 deaths expected during 2008 (1). Since colorectal cancers develop slowly over a long period, at least 10 years in most people (2), early intervention strategies such as a chemopreventive approach is an ideal choice to prevent colon cancer mortality.

Several studies from our laboratory and others have shown that combination of chemopreventive agents exerts greater efficacy than a single agent alone $(3,4)$. The combinational approaches are particularly significant when agents are combined at low-dose levels possessing diverse modes of action. Targeting different pathways of tumor cell survival may provide better efficacy and possibly without any side effects. In an earlier study, we have shown that a low-dose combination of celecoxib and lovastatin synergistically suppressed growth and induced apoptosis in HT-29 human cancer cells (5). Furthermore, studies using preclinical animal models carried out in our laboratory suggested that a combination of COX-1/2 inhibitor with HMG-R inhibitor provides synergistic colon tumor inhibitory effects (Proc Am Assoc Cancer Res, Abs. 2362, April, 2006). In studies with $\mathrm{APC}^{\mathrm{Min}}$ mice, a complete inhibition of colon tumor formation was observed using a combination of atorvastatin and celecoxib when compared to either agent alone (6). In another study, a low- dose of atorvastatin in combination with celecoxib and/or aspirin acted synergistically in suppressing azoxymethane-induced colon adenocarcinoma incidence and multiplicity in rats (7). Therefore, it is important to unravel the molecules and/or signaling pathways that play a role in exerting the combinational synergistic efficacy. This is particularly important given the concerns with life threatening side effects as observed in patients using high doses of the COX-2 selective inhibitor Rofecoxib. 
The identification of a particular cellular target modulated by a combination of statin and COX-2 inhibitors provides unique advantages for designing future chemopreventive strategies. We utilized a global gene array to identify several key targets that are modulated by a combination treatment of lovastatin and celecoxib in rat AOM-induced colon tumors. Based on the results from this experiment, we observed in rats fed a combination of $200 \mathrm{ppm}$ lovastatin and $300 \mathrm{ppm}$ celecoxib that the expression of caveolin-1 was more effectively suppressed than either agent administered alone (Proc Am Assoc Cancer Res, Abs. 2362, April, 2006). Caveolin-1 and cholesterol plays a crucial role in the formation of membrane microdomains such as lipid rafts and caveolae. Caveolin-1 contains a scaffolding domain to which a number of signaling molecules such as G-protein-coupled receptors, heterotrimeric $\mathrm{G}$ proteins, receptor tyrosine kinases, components of the Ras-mitogen-activated protein (MAP) kinase pathway, Src-like kinases, protein kinase C (PKC), nitric oxide synthase (NOS), H-Ras and eNOS are bound and concentrated (8-11). Previously, we have shown that caveolin-1 is overexpressed in rat colon tumors and is significantly associated with proliferative rates of human colon cancer cell lines (12). Caveolin-1 plays pivotal roles in intracellular signal transduction, angiogenesis and tumor invasion $(13,14)$. Therefore, we studied the effect of lovastatin and/or celecoxib on caveolin-1, Akt, Erk, Stat3, cyclin D1 and other cell cycle regulators. Excessive lipid body formation in cells associated with inflammation reactions have been reported $(15,16)$. Lipid bodies are spherical structures containing a mixture of triglycerides, eicosanoids and cholesterol esters encased in a thin phospholipids membrane (17). Since both cholesterol and caveolin-1 are essential for lipid body formation, we studied the effect of lovastatin, celecoxib and their combination on lipid body formation in human colon cancer cell lines.

In this study, we demonstrate for the first time that lovastatin and celecoxib both disrupt membrane caveolae and decrease the expression of Cav-1 associated signaling molecules. In particular, both drugs decreased phosphorylated AKT and its down-stream effector proteins. There was a significant suppression of lipid body formation by both lovastatin and celecoxib.

\section{Materials and methods}

Cell cultures. Human colon carcinoma cell line, HCT-116, was obtained from American Type Culture Collection. The cells were maintained in McCoys (Gibco-BRL, Grand Island, NY) supplemented with $10 \%$ FBS and $1 \%$ streptomycin and penicillin antibiotics at $37^{\circ} \mathrm{C}$ in a $5 \% \mathrm{Co}_{2}$ incubator. Cultures at $70-80 \%$ confluence were used for all experiments.

Cell viability assay. Cell viability was measured by 3-(4,5)-2, 5-diphenyltetrazolium bromide (MTT) assay. In brief, HCT-116 cells were grown in the presence or absence of various concentrations of celecoxib and lovastatin or a combination of both. After $24 \mathrm{~h}$, MTT reagent was added to the cell culture plate and incubated for $4 \mathrm{~h}$ at $37^{\circ} \mathrm{C}$. Subsequently, the culture plates were incubated overnight with $10 \%$ sodium dodecyl sulphate (SDS) solution. The absorbance at $540 \mathrm{~nm}$ was measured by a microtiter plate reader (BMG, Germany).

Immunofluroscence. HCT-116 cells were grown on six-well tissue culture plates. The cells were treated with $25 \mu \mathrm{M}$ lovastatin and/or $50 \mu \mathrm{M}$ celecoxib when they were $80 \%$ confluent. After $24 \mathrm{~h}$ incubation, the cultures were fixed in formalin. Immunofluorescence analysis was performed by tissue incubation in PBS containing 0.2\% saponin, 1\% BSA, followed by overnight incubation with Cav-1 (1:500; Santa Cruz Biotechnology Inc, USA) primary antibody at $4^{\circ} \mathrm{C}$. The goat anti-rabbit tagged to fluorescein (FITC) (Jackson Immunoresearch Laboratories, West Grove, PA, USA) was used for the secondary antibody. Following incubation with secondary antibody, nuclei were stained with $10 \mu \mathrm{g} / \mathrm{ml}$ DAPI for $5 \mathrm{~min}$. After each incubation step, the tissue was washed in PBS three times. Finally, the tissue was covered with aqueous mounting medium (Biomeda, CA) and examined under an Olympus IX71 fluorescent microscope.

Western blot analysis. Cells exposed to $25 \mu \mathrm{M}$ lovastatin and/or $50 \mu \mathrm{M}$ celecoxib were lysed in ice-cold lysis buffer (50 mM Tris pH 7.4, 150 mM NaCl, 1 mM EDTA, 1\% NP-40, $50 \mathrm{mM}$ NaF, $1 \mathrm{mM}$ sodium orthovanadate, $1 \mathrm{mM}$ PMSF, $1 \mathrm{mM}$ DTT and a protease inhibitor cocktail) and the protein content was quantitated using the Bio-Rad protein assay kit (Hercules, CA). Separation of proteins $(50 \mu \mathrm{g})$ was resolved on a SDSPAGE and transferred onto nitrocellulose membranes. Membranes were blocked with a solution containing $10 \mathrm{mM}$ Tris-HCl (pH 7.5), 150 mM/1 NaCl, 0.01\% (v/v) Tween-20 and $5 \%$ dry milk and incubated for $1 \mathrm{~h}$ with primary antibody (1:1000), anti-actin (1:1000). After washing the blots with TBST they were then incubated with anti-mouse and rabbit HRP-conjugated secondary antibody followed by washing again with TBST. Washed blots were incubated with Super Signal West Pico Chemiluminescence Substrate (Pierce, Rockford, IL) for $5 \mathrm{~min}$ and exposed to Kodak X-ray film.

Lipid body staining. HCT-116 cells were fixed in $4 \%$ formaldehyde and immersed in $1.0 \%$ thiocarbohydrazide for $5 \mathrm{~min}$, rinsed in $0.1 \mathrm{M}$ cacodylate buffer, restained with $1.5 \%$ $\mathrm{OsO}_{4}$ for $3 \mathrm{~min}$, rinsed in distilled water and then dried and mounted. Cell morphology was observed and lipid bodies were enumerated by microscopy.

\section{Results}

Growth inhibition of HCT-116 cells by lovastatin and celecoxib. To compare the growth inhibitory effect of HCT-116 cells in response to lovastatin and celecoxib, we used the MTT assay. In the MTT assay, thiazolyl blue (MTT) is reduced by mitochondrial dehydrogenases. The uptake of the vital dye MTT is a measure of the mitochondrial dehydrogenase activity within the cell and provides an indication of the proliferation status of the cells. The HCT-116 cell line was exposed to lovastatin $(0-100 \mu \mathrm{M})$ and celecoxib $(0-150 \mu \mathrm{M})$ for $24 \mathrm{~h}$. The $\mathrm{IC}_{50}$ value for HCT-116 cell growth inhibition was $25 \mu \mathrm{M}$ for lovastatin and $50 \mu \mathrm{M}$ for celecoxib. Hence we used $25 \mu \mathrm{M}$ lovastatin and $50 \mu \mathrm{M}$ celecoxib for all the experiments. 

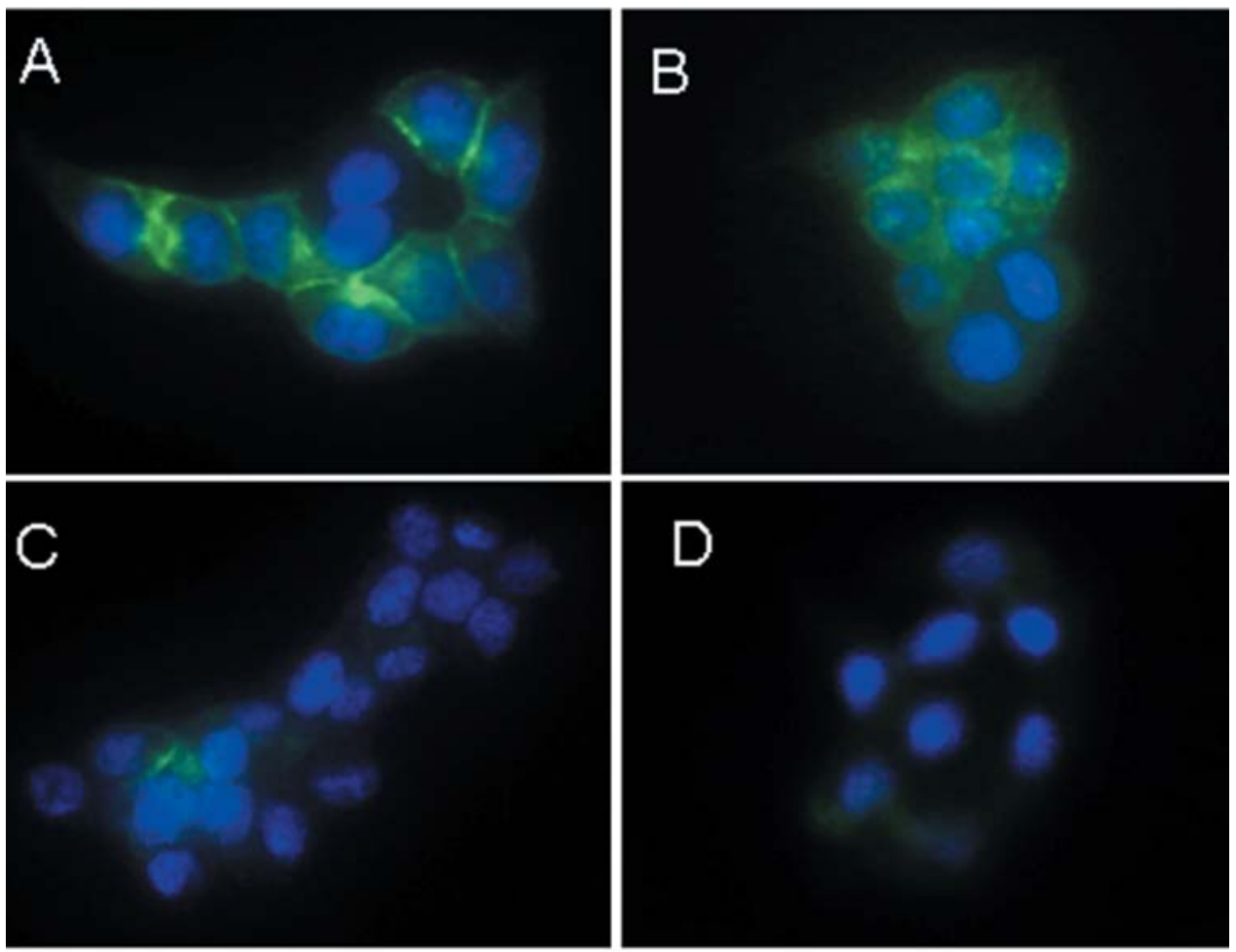

\section{E \\ Celecoxib} Lovastatin

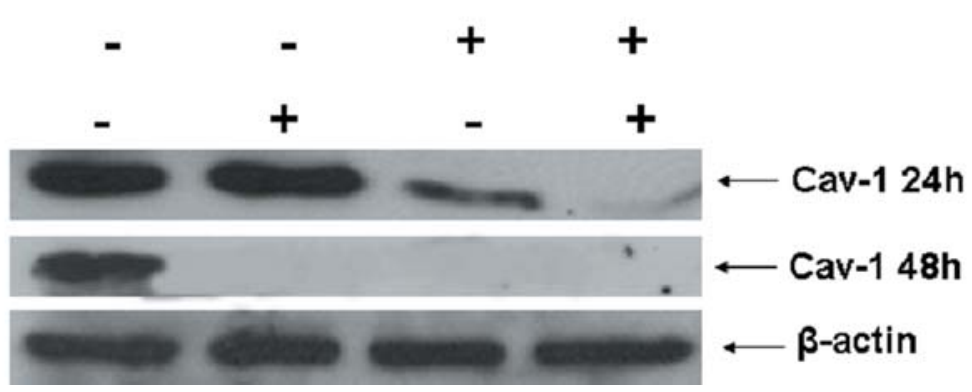

Figure 1. Effect of lovastatin and/or celecoxib on membrane caveolae. Following treatment of HCT-116 cells with $25 \mu \mathrm{M}$ lovastatin or $50 \mu \mathrm{M}$ celecoxib, immunofluorescence was performed to look for caveolin-1 expression (A) HCT-116 control (B) HCT-116 treated with $25 \mu \mathrm{M}$ lovastatin. (C) HCT-116 cells treated with $50 \mu \mathrm{M}$ celecoxib. (D) HCT-116 cells treated with lovastatin and celecoxib. (E) Western blot analysis of caveolin-1 expression from total cell lysates of HCT-116 after treatment with lovastatin, celecoxib and their combination for 24 and $48 \mathrm{~h}$, respectively.

Lovastatin and celecoxib decrease caveolin-1. The effect of lovastatin and celecoxib or a combination of both agents on caveolin-1 expression and localization is summarized in Fig. 1A-E. Cells exposed to celecoxib and its combination with lovastatin for $24 \mathrm{~h}$ showed a significant decrease in immunofluorescence of caveolin-1 protein (Fig. 1A-D). These results were further confirmed by Western blot analysis (Fig. 1E upper panel, $24 \mathrm{~h}$ treatment). While cells exposed to lovastatin for $24 \mathrm{~h}$ do not show any decrease in total immunofluoresence, it did lead to the disruption of membrane bound caveolin-1 into the cytoplasm (Fig. 1B). Notably, Western blot analysis of HCT-116 cells treated with lovastatin for $48 \mathrm{~h}$ showed a significant decrease of caveolin-1 protein (Fig. 1E, lower panel). Taken together, our results clearly suggest that the combination of lovastatin and celecoxib suppresses caveolin-1 expression more effectively than either agent alone (Fig. 1A-E).

Akt signaling is down-regulated by celecoxib and lovastatin. To understand whether molecules that are directly influenced by caveolin-1 are modulated by celecoxib and lovastatin, we investigated the effect of these agents on the AKT survival pathway. We examined the effect of lovastatin, celecoxib and their combination on EGF-induced AKT phosphorylation and its down-stream effector molecules. Our results show that, while total AKT levels are unaffected by either individual or combination treatment, both lovastatin and celecoxib decreased phosphorylation of AKT while the combination treatment showed total inhibition of AKT phosphorylation (Fig. 2). Similar to AKT phosphorylation, treatment of HCT-116 with lovastatin and/or celecoxib also decreased the levels of phosphorylated Erk1/2 (Fig. 2). Also, treatment with lovastatin and/or celecoxib at concentrations that block ERK-phosphorylation in HCT-116 cells inhibited STAT3 activation, indicating that the AKT-ERK-STAT3 pathway is involved in the proliferative effects of HCT-116 colon cancer cells (Fig. 2).

Effect of lovastatin and celecoxib on cell cycle and apoptosis proteins. Lovastatin and celecoxib showed a modest decrease in cyclin D1, while the combination treatment showed a robust 


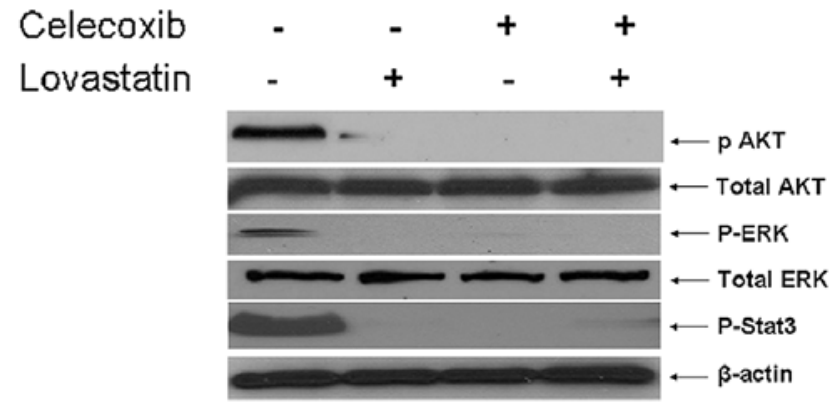

Figure 2. Inhibition of pAKT and its down-stream effectors in human colon cancer cells. HCT-116 cells were treated with lovastatin and/or celecoxib for 24 h. pAKT expression was stimulated by adding $5 \mathrm{nM}$ EGF for $5 \mathrm{~min}$. Western blots were carried out using total cellular proteins and membranes were probed with pAKT, AKT, p-ERK, total ERK and phospho-STAT3 antibodies.

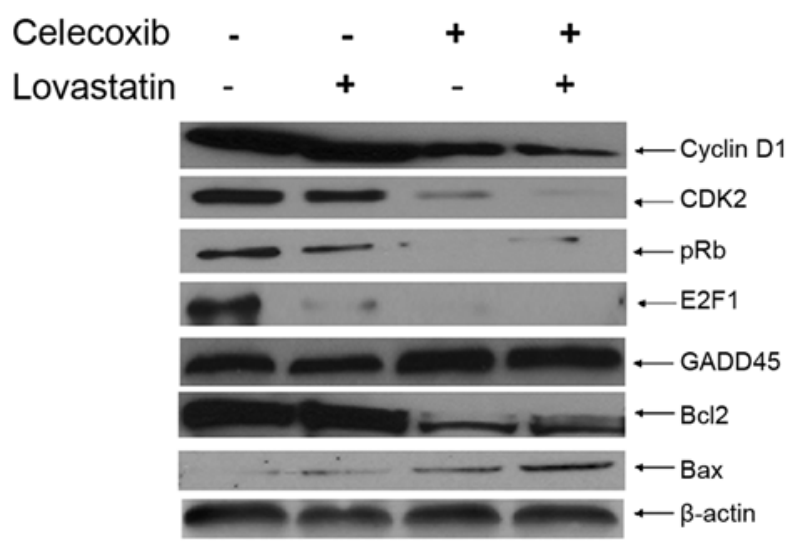

Figure 3. Inhibition of cell cycle and apoptosis proteins by lovastatin and/or celecoxib. Total cell lysates were obtained from HCT-116 cells after overnight treatment with lovastatin, celecoxib and their combination and analyzed for cyclin D1, CDK2, pRb, E2F1 GADD45, Bcl-2 and BAX was studied by Western blotting.

decrease, suggesting a synergistic effect (Fig. 3). Further, Western blot results revealed that lovastatin and celecoxib either alone or in combination decreased the expression of CDK2, pRb and E2F1 in HCT-116 colon cancer cells (Fig. 3). Although lovastatin treatment did suppress the expression of these proteins, the decrease was more pronounced in celecoxib and the combination treatment. With regard to apoptotic proteins, the expression of BAX showed a modest increase with lovastatin and celecoxib treatment, while there was a much higher increase in BAX expression in the combination treatment, a clear indication of a synergistic effect (Fig. 3). With regard to Bcl-2, only celecoxib and the combination treatment decreased its expression, while lovastatin had no effect (Fig. 3). Our studies on the expression of GADD45 showed that lovastatin and celecoxib showed little or no effect, while the combination treatment caused a modest increase in GADD45 (Fig. 3).

Lovastatin and celecoxib decrease lipid body formation. Lovastatin and/or celecoxib decreased the size as well as number of lipid bodies, which correlated well with the inhibitory effects of the compounds. As shown in Fig. 4, while there was a considerable amount of lipid bodies in control cells, there was a significant decrease in lipid body formation with lovastatin and/or celecoxib treatment. Lovastatin and celecoxib showed a $90 \%$ decrease in the amount of accumulated lipid bodies, while the combination treatment showed a complete inhibition.

\section{Discussion}

Previous studies from our laboratory have shown that combination of statins and/or celecoxib can reduce colon tumors in rodent models (6). However, the molecular mechanism by which synergistic inhibition occurs is not clearly known. In the present study, we show that lovastatin and celecoxib modulate caveolin-1 expression and thereby alter the signaling molecules associated with caveolin- 1 . The phosphorylation of AKT, a protein that is associated with the scaffolding domain of caveolin-1 promotes the activation of ERK and subsequently STAT3. We also show that lovastatin and/or celecoxib decreases cyclin D1, pRb and E2F1.

Caveolin-1 and its associated signaling molecules have been linked with cell proliferation, invasion, angiogenesis, metastasis, suppression of apoptosis and chemoresistance in several cancer types. There is evidence suggesting that caveolin-1 plays an important role in the promotion of colon tumorigenesis. In support of this, caveolin-1 is overexpressed in colon cancer cells $(18,12)$ and has been shown to promote cancer in an animal model of prostate cancer (19). In addition, caveolin-1 promotes cancer growth via inhibition of apoptosis through induction of cyclin D1 (20). In the current study, we observed that $24 \mathrm{~h}$ treatment of HCT-116 colon cancer cells with either celecoxib or the combination with lovastatin decreased caveolin-1 expression. Although overnight treatment with lovastatin did not decrease caveolin-1 expression, immunofluorescence studies showed that lovastatin induced membrane disassociation of caveolin-1 into the cytoplasm. In contrast, cells treated for $48 \mathrm{~h}$ with lovastatin completely decreased caveolin-1 expression. The reason for the above observations could be that lovastatin alters the membrane stability by blocking cholesterol biosynthesis, thereby releasing caveolin- 1 into the cytoplasm which is later degraded. However, the mechanism by which celecoxib decreases caveolin-1 is not yet understood.

The interaction between caveolin-1 and various signaling molecules that are bound to its scaffolding domain plays an important role in caveolin-1 mediated signaling involving growth and survival. Several signaling molecules such as Akt, G-protein-coupled receptors, heterotrimeric G proteins, receptor tyrosine kinases, components of the Ras-mitogenactivated protein (MAP) kinase pathway, Src-like kinases, protein kinase $\mathrm{C}$ (PKC), nitric oxide synthase (NOS), H-Ras and eNOS are bound and concentrated (8-11) and are known to bind to the scaffolding domain of caveolin-1. Akt (protein kinase B), a serine/threonine kinase, has emerged as a critical enzyme in several signal transduction pathways involved in cell proliferation, apoptosis, angiogenesis and diabetes. Increased Akt activity has been shown to be responsible for caveolin-1-mediated cell survival. Caveolin-1 has been shown to increase the half-life of phosphorylated PDK1 and Akt after inhibition of PI3-K by LY294002 (21). Indeed, many highly 

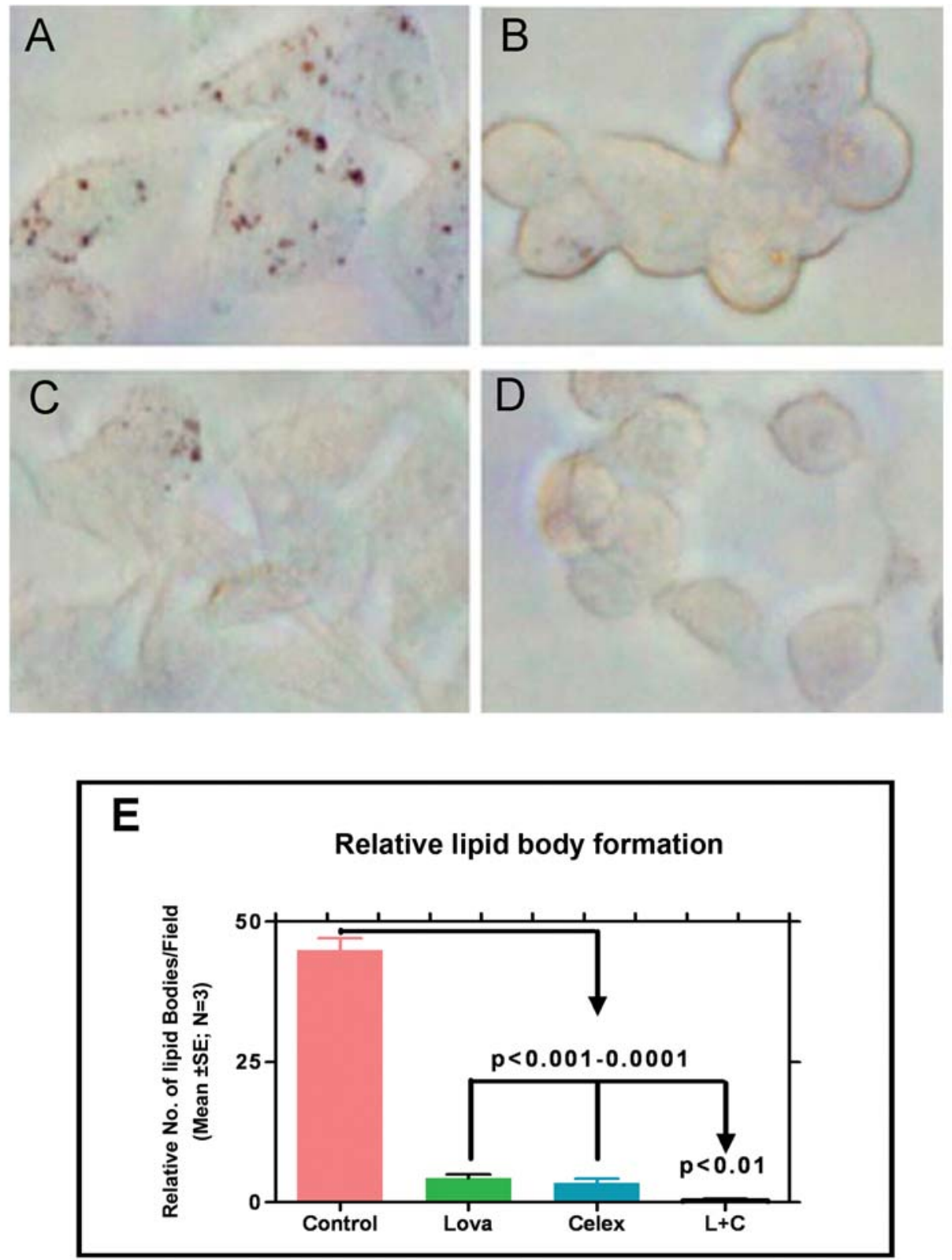

Figure 4. Effect of lovastatin and/or celecoxib on lipid body formation in HCT-116 cells. Cytoplasmic lipid bodies were evaluated in HCT-116 cells after overnight incubation with lovastatin, celecoxib and their combination. Lipid bodies were enumerated using light microscopy after staining with oil red O. (A) HCT-116 control (B) HCT-116 treated with $25 \mu \mathrm{M}$ lovastatin. (C) HCT-116 cells treated with $50 \mu \mathrm{M}$ celecoxib. (D) HCT-116 cells treated with lovastatin and celecoxib. (E) Statistical analysis of the relative number of lipid bodies between control and treated samples. Lova, lovastatin; celex, celecoxib and $\mathrm{L}+\mathrm{C}$, lovastatin + celecoxib.

metastatic cancer cells express high amounts of activated Akt (22). Activation of Akt occurs through phosphorylation at two regulatory residues, a threonine residue on the kinase domain and a serine residue on the hydrophobic motif (22). The principal role of Akt is to facilitate growth factor-mediated cell survival and block apoptotic cell death. Akt achieves this by phosphorylating a variety of substrates, including Erk, Bad, and STAT3 transcription factors (23-25). It therefore seems rational to hypothesize that lovastatin and celecoxib, by decreasing caveolin-1 expression, may also decrease the levels of the activated Akt protein. In agreement with this hypothesis, we found that both lovastatin and/or celecoxib decreased the phosphorylation of Akt.

The Erk signaling cascade is critical for transducting signals that are essential for differentiation, plasticity and survival
$(26,27)$. The Erk signaling cascade is activated by a wide variety of receptors involved in growth and differentiation such as receptor tyrosine kinases (RTKs). Activated Erk can regulate targets in the cytosol and also translocate to the nucleus where it phosphorylates a variety of transcription factors regulating expression of genes such as STAT3 (28). In this context, we studied the phosphorylation of ERK 1/2 after lovastatin and celecoxib treatment. Our results show that both lovastatin and celecoxib treatment led to a decrease in the phosphorylation of ERK1/2, which points to an inhibition of their kinase activity. The results from this study also show that both lovastatin and celecoxib decreased Bcl-2, while increasing BAX expression. Since Erk activation has been shown to be important for survival and is known to induce Bcl-2, the decrease in the phosphorylation of ERK observed in the 


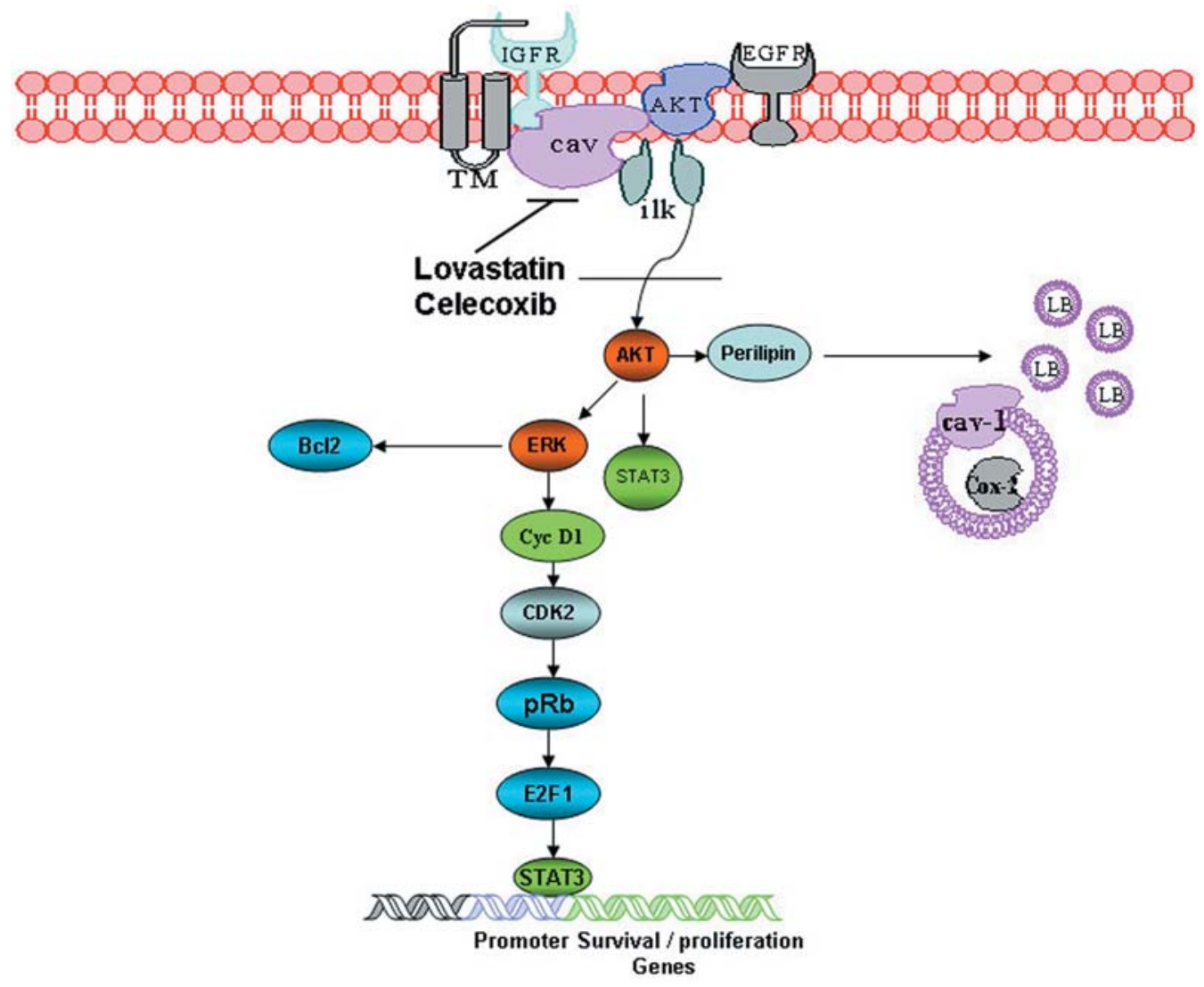

Figure 5. A model for lovastatin and celecoxib induced growth inhibition of human colon cancer cells. Lovastatin blocks cholesterol formation, thereby disrupting plasma membrane caveolae and caveolin-1-associated signaling. Several signaling molecules such as AKT, IGFR and Ilk are bound to caveolin-1. Celecoxib and lovastatin block AKT activation and subsequently ERK and STAT3. Down-regulation of ERK and STAT3 leads to down-regulation of genes involved in growth and proliferation such as cyclin D1, CDK2, pRb and E2F1. COX-2 and cholesterol promote lipid body formation. Lovastatin and celecoxib can effectively prevent its formation. Cyc D1, Cyclin D1; ERK, extracellular regulated kinase; AKT, also known as protein kinase B (PKB); STAT3, signal trandsducer and activator of transcription; Cav-1, caveolin-1; EGFR, epidermal growth factor receptor; IGFR, insulin growth factor receptor; TM, transmembrane protein; LB, lipid bodies and ilk, integrin linked kinase.

current study may be involved in the apoptotic induction by lovastatin and celecoxib.

Induction of cyclin D1 by growth factors and oncogenes is PI3'-kinase-dependent, which suggests that the PI3'-kinase/ Akt pathway may play an important role in the control of cell cycle progression $(29,30)$. Further, the ability of Akt to directly phosphorylate key mediators of the apoptotic response provides strong circumstantial evidence for the importance of the PI3'-kinase-Akt pathway in the aberrant behavior of cancer cells. The progression of cells into $S$ phase requires the activity of cyclin D1 (31). The ability of Akt to promote cell cycle progression is correlated with its ability to increase expression of cyclin D1. We show that celecoxib and/or lovastatin alters the levels of cyclin D1, and subsequently pRb and E2F1. Without active nuclear E2F-1 function, crucial S phase activities such as DNA repair are impaired, leading to eventual cell death. For instance, the toxicity of celecoxib has been shown to be in part through the down-stream inhibition of $\mathrm{pRb}$ and E2F-1 activity within the nucleus (32).

One important aspect of cholesterol regulation is intracellular cholesterol storage in lipid storage organelles, called lipid droplets or lipid bodies, which contain a pool of proteins with a wide range of biochemical activities. So far, only a few of these proteins have been identified and little is known about their structural properties and functions. High concentrations of lipid bodies develop in cells associated with inflammation reactions $(15,16)$. Various cytokines and pro-inflammatory stimuli trigger the synthesis of COX-2, leading to increased formation of eicosanoids. There is increasing evidence that lipid bodies are sites of intracellular localization of COX-2 $(33,34)$ as well as repositories of esterified arachidonates (35). Thus, lipid bodies serve as novel putative sites for eicosanoid biosynthesis in cells involved in inflammation (36-39). Caveolin-1 plays an important role in the modulation of lipolysis and lipid droplet formation (38). In support of this, caveolin-1 has been shown to be redirected from the plasma membrane caveolae to intracellular lipid droplets and mice lacking caveolin-1 have lean bodies with adipocyte abnormalities (38-40). Lipid bodies are sites at which key eicosanoid-forming enzymes are localized. The arachidonatereleasing enzyme $\mathrm{CPLA}_{2}$ and its activating MAP kinases also localizes to lipid bodies. PI3K has been shown to localize to lipid bodies of myeloid-derived cells, which would support a role for PI3K in generating phosphoinositide signaling molecules within lipid bodies. Therefore, it is likely that regulated signal transduction responses occur within lipid body domains. In this context, our findings that lovastatin and/or celecoxib inhibit lipid body formation are important for understanding the regulation of signaling molecules associated with lipid bodies (Fig. 5). Since lipid bodies contain cholesterol 
and COX-2 as well as caveolin-1, statins will be useful in disrupting the membrane integrity of the lipid bodies while COX-2 inhibitors can be used to block the activity of the COX-2 enzyme.

The increased expression of HMG-coA reductase and COX-2 are important pathological components in colon cancer. In this regard, our current findings provide further insights into the possible use of lovastatin and celecoxib in a low-dose combination for colon cancer chemoprevention and treatment.

\section{Acknowledgements}

We thank Wade Williams of the Oklahoma Cancer Institute for editorial help. This study was in part supported by R01CA-94962 and R01-CA-102947 from the National Cancer Institute (NCI).

\section{References}

1. American Cancer Society: Colorectal Cancer Facts. Atlanta, GA, USA, 2007

2. Smith R, von Eschenbach A and Wender R: American Cancer Society Guidlines for early detection of cancer: Update of early detection guidlines for prostate, colorectal and endometrial cancers. CA Cancer J Clin 51: 38-75, 2001.

3. Rao CV, Indranie C, Simi B, Manning PT, Connor JR and Reddy BS: Chemopreventive properties of a selective inducible nitric oxide synthase inhibitor in colon carcinogenesis, administered alone or in combination with celecoxib, a selective cyclooxygenase-2 inhibitor. Cancer Res 62: 165-170, 2002.

4. Swamy MV, Cooma I, Patlolla JM, Simi B, Reddy BS and Rao CV: Modulation of cyclooxygenase-2 activities by the combined action of celecoxib and decosahexaenoic acid: novel strategies for colon cancer prevention and treatment. Mol Cancer Ther 2: 215-221, 2004.

5. Swamy MV, Cooma I, Reddy BS and Rao CV: Lamin B, caspase-3 activity, and apoptosis induction by a combination of HMG-CoA reductase inhibitor and COX-2 inhibitors: a novel approach in developing effective chemopreventive regimens. Int J Oncol 20: 753-759, 2002

6. Swamy MV, Patlolla JM, Steele VE, Kopelovich L, Reddy BS and Rao CV: Chemoprevention of familial adenomatous polyposis by low doses of atorvastatin and celecoxib given individually and in combination to $\mathrm{APC}^{\mathrm{Min}}$ mice. Cancer Res 66: 7370-7377, 2006

7. Reddy BS, Wang CX, Kong AN, Khor TO, Zheng X, Steele VE, Kopelovich L and Rao CV: Prevention of azoxymethane-induced colon cancer by combination of low doses of atorvastatin, aspirin, and celecoxib in F 344 rats. Cancer Res 66: 4542-4546, 2006.

8. Anderson RGW: Caveolae: Where incoming and outgoing messengers meet. Proc Natl Acad Sci USA 90: 10909-10913, 1993.

9. Anderson RGW: The caveolae membrane system. Annu Rev Biochem 67: 199-225, 1998.

10. Li SW, Okamoto T, Chun MY, Sargiacomo M, Casanova JE, Hansen SH, Nishimoto I and Lisanti MP: Evidence for a regulated interaction between heterotrimeric $\mathrm{G}$ proteins and caveolin. J Biol Chem 270: 15693-15701, 1995.

11. Huang C, Hepler JR, Chen LT, Gilman AG, Anderson RG and Mumby SM: Organization of $G$ proteins and adenylyl cyclase at the plasma membrane. Mol Biol Cell 8: 2365-2378, 1997.

12. Patlolla JM, Swamy MV, Raju J and Rao CV: Overexpression of caveolin-1 in experimental colon adenocarcinomas and human colon cancer cell lines. Oncol Rep 11: 957-963, 2004

13. Simons K and Ikonen E: Functional rafts in cell membranes. Nature 387: 569-572, 1997.

14. Simons K and Toomre D: Lipid rafts and signal transduction. Nat Rev Mol Cell Biol 1: 31-39, 2001.

15. Coimbra A and Lopes-Vaz A: The presence of lipid droplets and the absence of stable sudanophilia in osmium-fixed human leukocytes. J Histochem Cytochem 19: 551-557, 1971.

16. Weinstein J: Synovial fluid leukocytosis associated with intracellular lipid inclusions. Arch Intern Med 140: 560-561, 1980.

17. Martin S and Parton RG: Lipid droplets: a unified view of a dynamic organelle. Nat Rev Mol Cell Biol 7: 373-378, 2006.
18. Fine SW, Lisanti MP, Galbiati F and Li M: Elevated expression of caveolin-1 in adenocarcinoma of the colon. Am J Clin Pathol 15: 719-724, 2001.

19. Yang G, Truong LD, Wheeler TM and Thompson TC: Caveolin-1 expression in clinically confined human prostate cancer: a novel prognostic marker. Cancer Res 59: 5719-5723, 1999.

20. Nagajyothi F, Desruisseaux M, Bouzahzah B, et al: Cyclin and caveolin expression in an acute model of murine Chagasic myocarditis. Cell Cycle 5: 107-112, 2006.

21. Li L, Ren CH, Tahir SA, Ren C and Thompson TC: Caveolin-1 maintains activated Akt in prostate cancer cells through scaffolding domain binding site interactions with and inhibition of serine/threonine protein phosphatases PP1 and PP2A. Mol Cell Biol 24: 9389-9404, 2003.

22. Bellacosa A, Kumar CC, Di Cristofano A and Testa JR: Activation of AKT kinases in cancer: implications for therapeutic targeting. Adv Cancer Res 94: 29-86, 2005.

23. Datta SR, Dudek H, Tao X, Masters S, Fu H, Gotoh Y and Greenberg ME: Akt phosphorylation of BAD couples survival signals to the cell-intrinsic death machinery. Cell 91: 231-241, 1997.

24. Kortylewski M, Feld F, Krüger KD, et al: Akt modulates STAT3-mediated gene expression through a FKHR (FOXO1a)dependent mechanism. J Biol Chem 278: 5242-5249, 2003.

25. Zhuang ZY, Xu H, Clapham DE and Ji RR: Phosphatidylinositol 3-kinase activates ER in primary sensory neurons and mediates inflammatory heat hyperalgesia through TRPV1 sensitization. J Neurosci 24: 8300-8309, 2004.

26. Sweatt JD: Mitogen-activated protein kinases in synaptic plasticity and memory. Curr Opin Neurobiol 14: 311-317, 2004

27. Wada T and Penninger JM: Mitogen-activated protein kinases in apoptosis regulation. Oncogene 23: 2838-2849, 2004

28. Kuroki $\mathrm{M}$ and O'Flaherty JT: Extracellular signal-regulated protein kinase (ERK)-dependent and ERK-independent pathways target STAT3 on serine-727 in human neutrophils stimulated by chemotactic factors and cytokines. Biochem J 341: 691-696, 1999.

29. Vanhaesebroeck B, Leevers SJ, Ahmadi K, et al: Synthesis and function of 3-phosphorylated inositol lipids. Annu Rev Biochem70: 535-602, 2001.

30. Fatrai S, Elghazi L, Balcazar N, Cras-Meneur C, Krits I, Kiyokawa $\mathrm{H}$ and Berna Mizrachi E: Akt induces beta-cell proliferation by regulating cyclin D1, cyclin D2, and p21 levels and cyclin-dependent kinase-4 activity. Diabetes 55: 318-325, 2006

31. Quelle DE, Ashmun RA, Shurtleff SA, Kato JY, Bar-Sagi D, Roussel MF and Sherr CJ: Overexpression of mouse D-type cyclins accelerates G1 phase in rodent fibroblasts. Genes Dev 7: $1559-1571,1993$

32. Bock JM, Menon SG, Sinclair LL, Bedford NS, Goswami PC, Domann FE and Trask DK: Celecoxib toxicity is cell cycle phase specific. Cancer Res 67: 3801-3808, 2007.

33. Dvorak AM, Morgan E, Tzizik DM and Weller PF: Prostaglandin endoperoxide synthase (cyclooxygenase): ultrastructural localization to nonmembrane-bound cytoplasmic lipid bodies in human eosinophils and murine 3T3 fibroblasts. Int Arch Allergy Immunol 105: 245-250, 1994.

34. Bozza PT, Yu W, Penrose JF, Morgan ES, Dvorak AM and Weller PF: Eosinophil lipid bodies: specific, inducible intracellular sites for enhanced eicosanoid formation. J Exp Med 186: 909-920, 1997.

35. Weller PF, Monahan-Earley RA, Dvorak HF and Dvorak AM: Cytoplasmic lipid bodies of human eosinophils: subcellular isolation and analysis of arachidonate incorporation. Am J Pathol 138: 141-148, 1991.

36. Bozza PT, Payne JL, Morham SG, Langenbach R, Smithies O and Weller PF: Leukocyte lipid body formation and eicosanoid generation: cyclooxygenase-independent inhibition by aspirin. Proc Natl Acad Sci USA 93: 11091-11096, 1996.

37. Serhan CN: Signalling the fat controller. Nature 384: 23-24, 1996.

38. Cohen AW, Razani B, Schubert W, et al: Role of caveolin-1 in the modulation of lipolysis and lipid droplet formation. Diabetes 53: 1261-1270, 2004

39. Fujimoto T, Kogo H, Ishiguro K, Tauchi $\mathrm{K}$ and Nomura R: Caveolin-2 is targeted to lipid droplets, a new 'membrane domain' in the cell. J Cell Biol 152: 1079-1085, 2001.

40. Razani B, Combs TP, Wang XB, et al: Caveolin-1-deficient mice are lean, resistant to diet-induced obesity, and show hypertriglyceridemia with adipocyte abnormalities. J Biol Chem 277: 8635-8647, 2002. 